\title{
Photooxidation of 9,10-Dimethylanthracene in Two Phase System: Benzene-Water
}

\author{
GUNNAR AKSNES and BERNT HENNING VAGSTAD
}

Chemical Institute, Realfagbygget, University of Bergen, N-5000 Bergen, Norway

The photooxidation of 9,10-dimethylanthracene dissolved in benzene and spread as liquid film on water is reported. The primary product is 9,10-dimethyl-9,10-anthracene endo-peroxide, which is further photodecomposed and hydrolysed by water to a series of oxygenated aromatic compounds. The rate of formation of the endoperoxide and its further photodecomposition are studied as functions of wave lengths of illumination.

The persistency of oil pollution at sea is at present hotly debated. ${ }^{1,2}$ Physical, chemical, and biochemical effects all influence the ultimate fate of the complex hydrocarbon mixture which constitutes the crude oil. An oil slick, which is spread on the sea surface is exposed to an enormous influx of solar energy. It is accordingly to be expected that photooxidation ought to play a considerable role in the degradation process. With reference to oil pollution, relatively few studies of photooxidation of the two phase system, Hydrocarbon - water, have been reported. ${ }^{2-7}$

One problem of special interest is the effect of different wave-lengths of radiation on the photooxidation process. This is expecially so since photooxidation of stable hydrocarbons is usually very slow outside the UV region, and the solar spectrum at the sea level contains almost no radiation below $300 \mathrm{~nm}$. On the other hand, it is known that olefins and various aromatic compounds are easily oxidised in the presence of singlet oxygen sensitizers, such as methylene blue, rose bengal, and other dyes, porphyrins and various polycyclic aromatic compounds. $^{8-15}$ Photosensitizers of singlet oxygen are effective in the visible solar spec- trum due to the low activation energy necessary for triplet-singlet transformation of oxygen (approx. $\left.96 \mathrm{~kJ} \quad \mathrm{~mol}^{-1}\right) .^{8} \quad$ Phorphyrins and polycyclic aromatic compounds, both alkylated and hydroxylated, are present in crude oils in considerable amounts. ?

Photooxidation studies of anthracenes in homogeneous solution have been reported to give endoperoxides and several secondary photodecomposition products. Photochemical ${ }^{16}$ as well as acid catalysed ${ }^{17}$ decomposition of 9,10-anthracene endoperoxides give rise to 9,10-dihydroxy derivatives, 9-hydroxy anthrones, anthraquinone, a ketal of structure 1 , and a cyclobutane 1,4-dioxane derivative 2 .
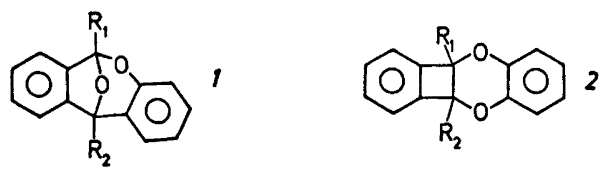

In the present paper we have studied the effect of varying the wave lengths of radiation during air oxidation of a benzene solution of 9,10-dimethylanthracene (DMA) spread on water. We have been especially interested in investigating the rate and distribution of the main oxidation products as function of wave lengths of light and time of illumination. Photochemical formation of endoperoxide, and the light induced decomposition of the endoperoxide have therefore been studied separately in order to clarify the dynamic behaviour of the overall oxidation process. 


\section{EXPERIMENTAL}

The illumination experiments were performed in a circular reaction vessel of $100 \mathrm{~mm}$ diameter containing $250 \mathrm{ml}$ of water. The hydrocarbon solution was floating on the water surface in a $4 \mathrm{~mm}$ thick layer. The temperature was kept at $7 \pm 1^{\circ} \mathrm{C}$. The hydrocarbon solution was illuminated by a $100 \mathrm{~W}$ mercury medium pressure lamp (Hanovia type) placed vertically $30 \mathrm{~cm}$ above the surface.

The wave length of the radiation was varied by using a circular edge filter of $100 \mathrm{~mm}$ diameter and $2 \mathrm{~mm}$ thickness (Schott-Jena): WG 230 ("218"), WG 305 ("300"), WG 345 ("341"), GG 395 ("388"), and GG 420 ("417"). The numbers within parantheses refer to the wave length at which half of the light intensity is transmitted. In the following the filters will be referred to according to the latter numbers.

Product analysis was performed with a high pressure liquid chromatograph (HPLC), Du Pont, Model 830, with a variable wave length UV detector, fixed at $236 \mathrm{~nm}$. The conditions were: ODS reversed phase column, pressure 12.4 MPa, mobile phase: $\mathrm{MeOH} / \mathrm{H}_{2} \mathrm{O}$ 60:40.

Preparation of 9,10-dimethyl-9,10-anthracene endo-peroxide $\left(\mathrm{DMA}-\mathrm{O}_{2}\right) .^{18}$ 9,10-Dimethyl anthracene (DMA, $380 \mathrm{mg}$ ) was dissolved in $240 \mathrm{ml}$ of $\mathrm{CH}_{2} \mathrm{Cl}_{2}$. To this solution was added a mixture of $49 \mathrm{mg}$ of 18-crown-6 and $49 \mathrm{mg}$ of rose bengal dissolved in $50 \mathrm{ml}$ of $\mathrm{CH}_{2} \mathrm{Cl}_{2}$. A gentle stream of oxygen was bubbled through the solution during illumination for $2 \mathrm{~h}$ using filter " 300 ". The solution was then filtered through a neutral alumina column, and the solvent evaporated. The yield of DMA-O $\mathrm{O}_{2}$ was $410 \mathrm{mg}(93 \%)$. HPLC as well as NMR analysis showed a pure product.

1,2-Diacetylbenzene was prepared by $\mathrm{CrO}_{3}$ oxidation of 1,4-dimethylnaphthalene according to literature procedure. ${ }^{19}$

Photodegradation. (a) 9,10-Dimethylanthracene (DMA). DMA $(27.5 \mathrm{mg}, 0.13 \mathrm{mmol})$ was dissolved in $30 \mathrm{ml}$ of benzene. Biphenyl (5.2 mg) was added as internal standard. The benzene solution was poured on the surface of $250 \mathrm{ml}$ of water in the above reaction vessel. A gentle stream of oxygen was bubbled into the water layer during the illumination. Samples were withdrawn at different intervals, the benzene evaporated and the rest dissolved in $\mathrm{CH}_{2} \mathrm{Cl}_{2}$ and analysed on HPLC. Three classes of products were separated, a strongly polar group of compounds (SP) were eluted just after the solvent, followed by $\mathrm{DMA}-\mathrm{O}_{2}$, and a last peak consisting of a medium polar group of compounds (MP). The peaks had the following relative retention times: $\mathrm{SP}: 0.33$, DMA-O $\mathrm{O}_{2}$ : 0.43 , MP: 0.63 , biphenyl: 1.00, DMA: 5.74. The products were separated preparatively on TLC plates using silica gel and $2.5 \% \mathrm{MeOH}$ in chloroform as mobile phase. The isolated bands were analysed by MS. The filters used in the illumination were " 218 ", “300", "388", and "417". (b) 9,10-Dimethyl-9,10-anthracene endoperoxide $\left(D M A-O_{2}\right)$. DMA-O $(31.7 \mathrm{mg}, 0.13 \mathrm{mmol})$ was dissolved in $30 \mathrm{ml}$ of benzene and biphenyl $(5.2 \mathrm{mg})$ were added as internal standard. The solution was illuminated in the same way as in the previous experiment. The following filters were used: "218", "300", "341", and "388".

Quantitative measurements. The various products were determined quantitatively on HPLC by taking the ratio between the peak of the added internal standard, biphenyl, and the product. Calibration for DMA and DMA-O showed that the ratio between the peak heights was proportional to the concentration.

\section{RESULTS AND DISCUSSION}

Figs. 1-4 summarize the different illumination experiments with DMA and DMA-O where wave lengths and time of illumination have been varied. In all figures the $y$-axis gives the peak height ratio between the actual compound and the internal standard.

On GLC the MP-fraction from HPLC was found to contain two compounds, in approximately equal amounts. The first compound was identified as anthraquinone. The second compound had molecular weight 238, being also the base peak in the mass spectrum. Additional significant peaks were: $m / \mathrm{e} 223$ (38\%), 220 (18\%), 207 (31\%), 195 (96\%). NMR spectrum showed two singlets at $\delta 2.01$ and 2.11, respectively, in addition to the aromatic multiplet between 7.10 and 7.50. The data confirm that the compound is the earlier described ketal 1.

Anthraquinone and ketal 1 amount to $80-$ $90 \%$ of the total products in the experiments with DMA as well as DMA-O ${ }_{2}$ when filters

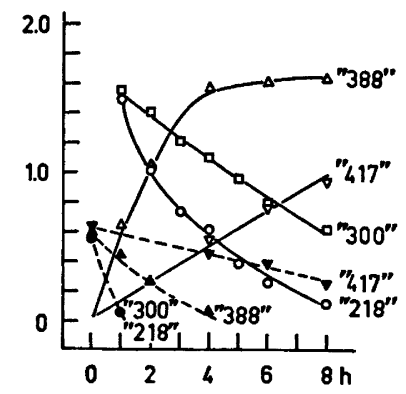

Fig. 1. Rate of disappearance of DMA (-.) and formation of $\mathrm{DMA}-\mathrm{O}_{2}(-)$ on illumination of DMA in benzene at various wave lengths. 


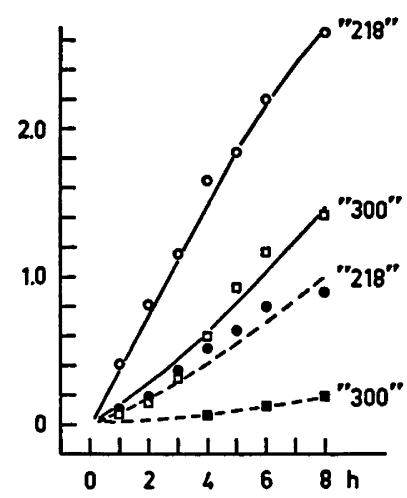

Fig. 2. Rate of formation of oxidation products "SP" (-.) and "MP" (-) on illumination of DMA in benzene.

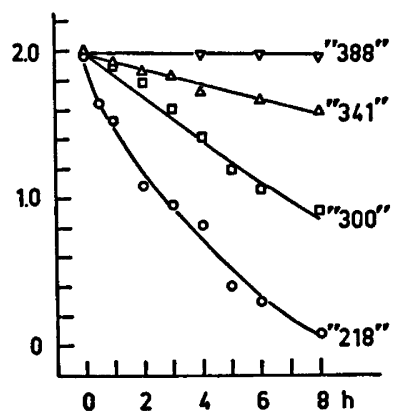

Fig. 3. Rate of disappearance of $\mathrm{DMA}-\mathrm{O}_{2}$ on illumination of its benzene solution at various wave lengths.

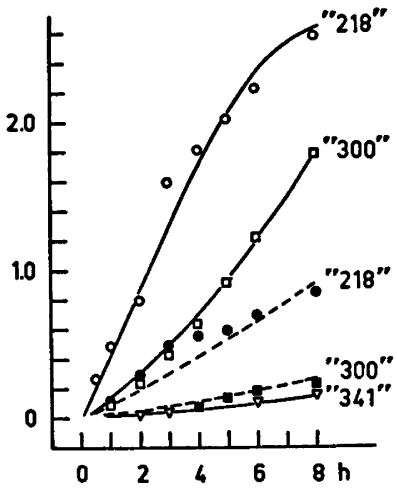

Fig. 4. Rate of formation of "SP" (-..) and "MP" $(-)$ on illumination of DMA in benzene.
" 218 " or " 300 " were used during the illumination. In the experiments with DMA the hydrocarbon was totally consumed in about $1 \mathrm{~h}$ when filters " 218 " as well as " 300 " were used. The reaction could be followed visually by the decoloration of the originally yellow solution of DMA. In both experiments DMA. $\mathrm{O}_{2}$ was found almost exclusively after $1 \mathrm{~h}$, but its concentration diminished quickly when filter "218" was used, somewhat slower with filter " 300 ". However, in both cases there was no endoperoxide left after illumination for $24 \mathrm{~h}$. With filter " 218 " there was also significantly more of the strongly polar compounds (SP) than with filter " 300 ", resulting in a yellowish water layer.

In the illumination experiments with filters " 388 " and " 417 " the rate of DMA oxidation was much slower, and the only product was DMA $-\mathrm{O}_{2}$, which appeared to be stable at these wave lengths. With filter " 388 " all DMA had disappeared after about $5 \mathrm{~h}$ during which the DMA. $\mathrm{O}_{2}$ concentration had reached a constant level. With filter " 417 " the DMA concentration was about $30 \%$ of the original after illumination for $8 \mathrm{~h}$. Illumination of $\mathrm{DMA}-\mathrm{O}_{2}$ under the same light conditions gave parallel results, but the concentration of DMA- $\mathrm{O}_{2}$ as well as of the various decomposition products was always higher after the same illumination time.

All of these experiments indicate that photooxidation of DMA in a hydrocarbon film on water proceeds through addition of singlet oxygen to DMA forming an endoperoxide (DMA-O $)_{2}$ ). The addition is quite rapid when light of shorter wave lengths than $388 \mathrm{~nm}$ is used, but even at $417 \mathrm{~nm}$ considerable oxidation of DMA takes place. Knowing that the longest wave length UV-band of DMA has its maximum at $395 \mathrm{~nm}$ with a long tail extending into the visible region (lg $\varepsilon \approx 2.4$ at $420 \mathrm{~nm}$ ) there seems to be a close connection between the placing of the UV-band of DMA and the ability to react with singlet oxygen. DMA must absorb light in order to transform triplet oxygen to singlet oxygen before addition of ${ }^{1} \mathrm{O}_{2}$ to DMA can take place.

A similar connection between UV-absorption and photochemical activity seems to exist for the decomposition of the endoperoxide formed. The longest wave length UV-band of DMA-O has its maximum at $278 \mathrm{~nm}$ with a tail extending

Acta Chem. Scand. B 33 (1979) No. 1 
to around $300 \mathrm{~nm}$. Since the edge filters used do not stop the light sharply at the edge but will also have transmission tails, it is reasonable that experiments with filter " 300 " as well as "341" will still give small amounts of decomposition products. The endoperoxide bridge in the anthracene molecule destroys the conjugation in the molecule resulting in a $100 \mathrm{~nm}$ displacement of the absorption maximum towards shorter wave length. There will accordingly exist a region in the spectrum between 300 and $400 \mathrm{~nm}$, where the DMA $-\mathrm{O}_{2}$ rapidly accumulates without being photochemically decomposed.

The strongly polar fraction (SP) consists of several compounds partly separated on TLC. The structures of these compounds are inferred from their MS fragmentations, but confirmation of their identities requires further physical and chemical evidence. With increasing $R_{F}$ values compounds with the following molecular weights were found in MS; 256 (3), 254 (4), $252(5), 240(6), 224(7)$, and $162(8)$.

The mass spectrum of 8 has characteristic peaks at $m / e 147$ (100\%), 105 (13\%), 91 (47\%), $43(53 \%)$. This spectrum fits well with the mass spectrum of an authentic sample of 1,2-diacetylbenzene, which is assumed to be formed from the endoperoxide through the following mech. anism;

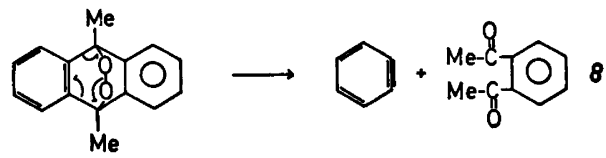

The molecular weights of $3-7$ show that the compounds have two or three oxygens, and the character of the mass spectrum indicates that they have retained the three-ring anthracene structure.

The mass spectra of 4 and 5 both show strong M-45 peaks, base peak in 4, and $44 \%$ of base peak intensity in 5 , indicating the presence of a $\mathrm{COOH}$ group, that may be obtained by oxidation of one of the $\mathrm{CH}_{3}$ groups. Compound 5 also has prominent $M-28$ and $M-29$ (base) peaks characteristic for a phenolic $\mathrm{OH}$ group.

The compound 6 with molecular weight $240(67 \%$ of base peak intensity in MS) has prominent peaks at $m / e 225$ (48 \%), $223(100 \%)$ and $208(79 \%)$, and a reasonable compound wlli be the 9,10-dihydroxy derivative of DMA.
The mass fragmentation ion at $m / e \quad 225$ $(\mathrm{M}-31)$ in 3 indicates the presence of an $\mathrm{OCH}_{3}$ group; a compound with a hemiketal structure is suggested.

The compound 7 with prominent peaks at $m / e 209(M-15)$ and $207(M-17)$ may be an anthrone derivative, that can be assumed to arise from the hydrolysis of 3.

According to this the reaction scheme for the various products can be imagined as follows:

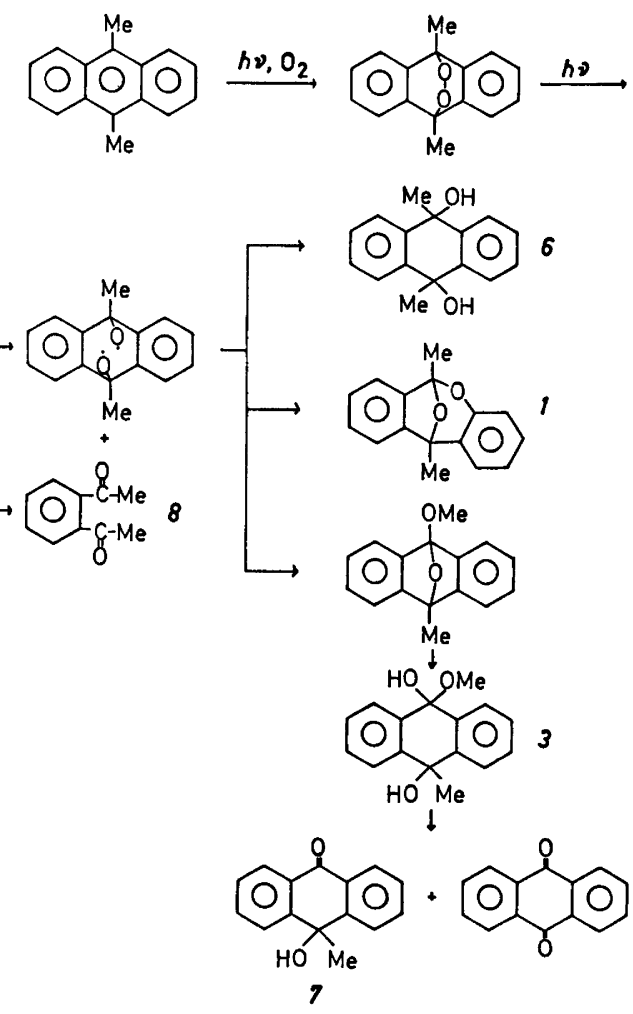

The present work has been supported by The Norwegian Research Council for Science and Humanities (NAVF) and the Marine Pollution Research and Monitoring Programme (FOH).

\section{REFERENCES}

1. The Fate and Effect of Oil in the Sea, American Petroleum Institute, Washington D.C. 1978.

2. Burwood, R. and Speers, G. C. Adv. Org. Geochem. (1975) 1005.

3. Hansen, H. P. Marine Chem. 3. (1975) 183.

4. Nagata, S. and Kondo, G. Oil Spill Conference, New Orleans 1977, p. 617.

Acta Chem. Scand. B 33 (1979) No. 1 
5. Larson, R. A., Hunt, L. L. and Blockenship, D. W. Environ. Sci. Technol. 5 (1977) 491.

6. Burwood, R. and Speers, G. C. Estuarine Coastal Mar. Sci. 2 (1974) 117.

7. Klein, A. E. and Pilpel, N. Water Res. 8 (1974) 79 .

8. Foote, C. S. Acc. Chem. Res. 1 (1968) 104.

9. Nagy, B. and Colombo, U., Eds., Fundamental aspects of petroleum geochemistry, Elsevier, New York and Amsterdam 1967.

10. Kahn, A. U. J. Phys. Chem. 80 (1976) 2219.

11. Stevens, B., Perez, S. R. and Ors, J. A. J. Am. Chem. Soc. 96 (1974) 6846.

12. Winters, K. and Parker, P. L. Oil Spill Conference, New Orleans 1977, p. 579.

13. Rigaudy, J. Pure Appl. Chem. (1968) 169.

14. Rigaudy, J. Actualité Chimique (1974) 7.

15. Kearns, D. R. and Merkel, P. B. J. Am. Chem. Soc. 94 (1972) 7244.

16. Rigaudy, J. and Breliere, C. Bull. Soc. Chim. Fr. (1972) 1390.

17. Rigaudy, J., Breliere, C. and Scribe, P. Tetrahedron Lett. (1978) 687.

18. Boden, R. M. Synthesis (1975) 783.

19. Riemscheider, R. and Foenster, S. Monatsh. Chem. 93 (1962) 616.

Received August 21, 1978.

Acta Chem. Scand. B 33 (1979) No. 1 